\title{
Nontrivial Solutions for a Boundary Value Problem of $n$ th-Order Impulsive Differential Equation
}

\author{
Jiafa $\mathrm{Xu}^{1}$ and Zhongli Wei ${ }^{1,2}$ \\ ${ }^{1}$ School of Mathematics, Shandong University, Jinan, Shandong 250100, China \\ ${ }^{2}$ Department of Mathematics, Shandong Jianzhu University, Jinan, Shandong 250101, China \\ Correspondence should be addressed to Jiafa Xu; xujiafa292@sina.com
}

Received 3 October 2013; Accepted 28 November 2013; Published 23 January 2014

Academic Editors: H. Lin and D.-B. Wang

Copyright (c) $2014 \mathrm{~J}$. Xu and Z. Wei. This is an open access article distributed under the Creative Commons Attribution License, which permits unrestricted use, distribution, and reproduction in any medium, provided the original work is properly cited.

We study the existence of nontrivial solutions for $n$ th-order boundary value problem with impulsive effects. We utilize LeraySchauder degree theory to establish our main results. Furthermore, our nonlinear term $f$ is allowed to grow superlinearly and sublinearly.

\section{Introduction}

In this paper, we consider the existence of nontrivial solutions for the following $n$ th-order boundary value problem involving impulsive effects:

$$
\begin{gathered}
u^{(n)}(t)+f(t, u(t))=0, \quad t \in[0,1], t \neq t_{k}, \\
-\left.\Delta u^{(n-1)}\right|_{t=t_{k}}=I_{k}\left(u\left(t_{k}\right)\right), \quad k=1,2, \ldots, m, \\
u(0)=\int_{0}^{1} u(t) \mathrm{d} \alpha(t), u(1)=\int_{0}^{1} u(t) \mathrm{d} \beta(t), \\
u^{\prime}(0)=\cdots=u^{(n-3)}(0)=u^{(n-2)}(0)=0,
\end{gathered}
$$

where $f \in C([0,1] \times \mathbb{R}, \mathbb{R}), I_{k} \in C\left(\mathbb{R}, \mathbb{R}^{+}\right), \int_{0}^{1} u(t) \mathrm{d} \alpha(t)$ and $\int_{0}^{1} u(t) \mathrm{d} \beta(t)$ denote the Riemann-Stieltjes integral, and $\alpha$ and $\beta$ are right continuous on $[0,1)$, left continuous at $t=1$, and nondecreasing on $[0,1]$, with $\alpha(0)=\beta(0)=0$.

The theory of impulsive differential equations describes processes which experience a sudden change of their state at certain moments. Therefore, many research workers pay their attention to boundary value problems for impulsive differential equations; for example, see [1-10] and the references therein.

In [1], D. Guo utilized fixed point theory to establish some existence theorems of positive solutions for an infinite boundary value problem of $n$ th-order nonlinear impulsive singular integrodifferential equations on the half-line in Banach spaces.

In $[2,3]$, he dealt with multiple positive solutions for the following infinite boundary value problem of first order impulsive superlinear integrodifferential equations on the half-line:

$$
\begin{gathered}
u^{\prime}(t)=f(t, u(t),(T u)(t),(S u)(t)), \quad \forall t \in J_{+}^{\prime}, \\
\left.\Delta u\right|_{t=t_{k}}=I_{k}\left(u\left(t_{k}\right)\right), \quad k=1,2,3, \ldots, u(\infty)=\beta u(0) .
\end{gathered}
$$

In [2], the function $f$ is continuous, that is, no singularity, but in [3] the author discussed the singular case, that is, $f \rightarrow \infty$ as $t \rightarrow 0^{+}$and $u \rightarrow 0^{+}$. Compare with [2], the key point of [3] is apart from the singular point $u=0$ by some appropriate functions.

Motivated by the works cited above, in particular [1-3], in this paper, we utilize the methods in [11] to study the existence of nontrivial solutions for (1). Nevertheless, our work here improves and extends the corresponding ones in [11]. We first note the impulsive effect as a perturbation to the corresponding problem of (1) without impulse, so we can construct an integral operator for the corresponding boundary value problem without the impulsive terms and find out its first eigenvalue and eigenfunction. Then we establish a special cone associated with the Green function of 
(1). Finally, by employing Leray-Schauder degree theory, two existence theorems of nontrivial solutions for (1) are obtained.

\section{Preliminaries}

We first offer some related preliminaries used in the ensuing section. In this work, we always assume that the following two conditions are satisfied:

(H1) $f \in C([0,1] \times \mathbb{R}, \mathbb{R}), I_{k} \in C\left(\mathbb{R}, \mathbb{R}^{+}\right)$,

$(\mathrm{H} 2) \kappa_{1} \geq 0, \kappa_{4} \geq 0, \kappa:=\kappa_{1} \kappa_{4}-\kappa_{2} \kappa_{3}>0$, where

$$
\begin{gathered}
\kappa_{1}:=1-\int_{0}^{1}\left(1-t^{n-1}\right) \mathrm{d} \alpha(t), \quad \kappa_{2}:=\int_{0}^{1} t^{n-1} \mathrm{~d} \alpha(t), \\
\kappa_{3}:=\int_{0}^{1}\left(1-t^{n-1}\right) \mathrm{d} \beta(t), \quad \kappa_{4}:=1-\int_{0}^{1} t^{n-1} \mathrm{~d} \beta(t) .
\end{gathered}
$$

For any $g \in C[0,1]$, the boundary value problem

$$
\begin{gathered}
u^{(n)}(t)+g(t)=0, \quad t \in[0,1], \\
u(0)=u^{\prime}(0)=\cdots=u^{(n-3)}(0)=u^{(n-2)}(0)=u(1)=0,
\end{gathered}
$$

if and only if $u$ can be expressed by $u(t)=\int_{0}^{1} H(t, s) g(s) \mathrm{d} s$, where

$$
\begin{aligned}
& H(t, s) \\
& =\frac{1}{(n-1) !} \begin{cases}(1-s)^{n-1} t^{n-1}-(t-s)^{n-1}, & 0 \leq s \leq t \leq 1, \\
(1-s)^{n-1} t^{n-1}, & 0 \leq t \leq s \leq 1 .\end{cases}
\end{aligned}
$$

Lemma 1 (see [12], Lemma 2.1). $H(t, s)$ has the following properties:

(i) $0 \leq H(t, s) \leq K(s), \forall t, s \in[0,1]$, where $K(s):=$ $s(1-s)^{n-1} /(n-2)$ !,

(ii) $H(t, s) \geq \zeta(t) K(s), \forall t, s \in[0,1]$, where $\zeta(t):=1 /(n-$ 1) $\min \left\{t^{n-1},(1-t) t^{n-2}\right\}$.

Let $E:=C[0,1],\|u\|:=\max _{t \in[0,1]}|u(t)|$, and $P:=\{u \in E$ : $u(t) \geq 0, \forall t \in[0,1]\}$. Then $(E,\|\cdot\|)$ is a real Banach space and $P$ is a cone on $E$. Set $J:=[0,1], J^{\prime}=[0,1] \backslash\left\{t_{1}, t_{2}, \ldots, t_{m}\right\}$ and introduce the following space:

$$
\begin{aligned}
P C^{n-1}[0,1]:= & \left\{u \in C[0,1]:\left.u^{(n-1)}\right|_{\left(t_{k}, t_{k+1}\right)} \in C\left(t_{k}, t_{k+1}\right),\right. \\
& \left.u^{(n-1)}\left(t_{k}^{-}\right)=u^{(n-1)}\left(t_{k}\right), \exists u^{(n-1)}\left(t_{k}^{+}\right)\right\}, \\
& k=1,2, \ldots, m,
\end{aligned}
$$

with the norm $\|u\|_{P C^{n-1}}:=\max \left\{\|u\|,\left\|u^{\prime}\right\|, \ldots,\left\|u^{(n-1)}\right\|\right\}$. Clearly, $\left(P C^{n-1}[0,1],\|\cdot\|_{P C^{n-1}}\right)$ is also a real Banach space.
Lemma 2 (see [9], Lemma 2.1). Let (H1) and (H2) hold. Then (1) is equivalent to the following integral equation:

$$
u(t):=\int_{0}^{1} G(t, s) f(s, u(s)) d s+\sum_{k=1}^{m} G\left(t, t_{k}\right) I_{k}\left(u\left(t_{k}\right)\right),
$$

where

$$
\begin{aligned}
& G(t, s) \\
& =H(t, s)+\kappa^{-1}\left(\kappa_{4}\left(1-t^{n-1}\right)+\kappa_{3} t^{n-1}\right) \int_{0}^{1} H(t, s) d \alpha(t) \\
& \quad+\kappa^{-1}\left(\kappa_{2}\left(1-t^{n-1}\right)+\kappa_{1} t^{n-1}\right) \int_{0}^{1} H(t, s) d \beta(t)
\end{aligned}
$$

$$
\begin{aligned}
& \text { Let } \eta(t):=\min \left\{t^{n-1}, 1-t^{n-1}\right\} \text { and } \\
& \qquad \gamma(t):=\min \left\{\zeta(t), \eta(t) \int_{0}^{1} \zeta(t) d \alpha(t)(\alpha(1))^{-1},\right. \\
& \left.\eta(t) \int_{0}^{1} \zeta(t) d \beta(t)(\beta(1))^{-1}\right\} .
\end{aligned}
$$

Here, $\zeta(t)$ is determined by Lemma 1. Clearly, $\gamma(t)$ is nonnegative and continuous on $[0,1]$.

Lemma 3. One has $G(t, s) \geq \gamma(t) G(\tau, s), \forall t, s, \tau \in[0,1]$.

Proof. By Lemma 1 and (8), we obtain $G(t, s) \leq K(s)+$ $\kappa^{-1} \alpha(1)\left(\kappa_{4}+\kappa_{3}\right) K(s)+\kappa^{-1} \beta(1)\left(\kappa_{2}+\kappa_{1}\right) K(s):=\mathscr{F}_{1}(s)$, and

$G(t, s)$

$$
\begin{aligned}
& \geq \zeta(t) K(s)+\kappa^{-1}\left(\kappa_{4}\left(1-t^{n-1}\right)+\kappa_{3} t^{n-1}\right) \\
& \quad \times \int_{0}^{1} \zeta(t) \mathrm{d} \alpha(t) K(s) \\
& \quad+\kappa^{-1}\left(\kappa_{2}\left(1-t^{n-1}\right)+\kappa_{1} t^{n-1}\right) \int_{0}^{1} \zeta(t) \mathrm{d} \beta(t) K(s) \\
& \geq \gamma(t)\left[K(s)+\kappa^{-1} \alpha(1)\left(\kappa_{4}+\kappa_{3}\right) K(s)\right. \\
& \left.\quad+\kappa^{-1} \beta(1)\left(\kappa_{2}+\kappa_{1}\right) K(s)\right] \\
& =\gamma(t) \mathscr{F}_{1}(s) .
\end{aligned}
$$

Combining these, we can find $G(t, s) \geq \gamma(t) \mathscr{F}_{1}(s) \geq$ $\gamma(t) G(\tau, s), \forall t, s, \tau \in[0,1]$. This completes the proof.

Let

$(A u)(t):=\int_{0}^{1} G(t, s) f(s, u(s)) \mathrm{d} s+\sum_{k=1}^{m} G\left(t, t_{k}\right) I_{k}\left(u\left(t_{k}\right)\right)$,

$(L u)(t):=\int_{0}^{1} G(t, s) u(s) \mathrm{d} s, \quad u \in E$. 
Clearly, $A: E \rightarrow E$ is a completely continuous nonlinear operator, and the existence of the solutions of (1) is equivalent to that of fixed points of $A ; L: E \rightarrow E$ is a completely continuous linear operator, satisfying $L(P) \subset P$. Namely, $L$ is a positive, completely continuous, linear operator. By the positivity of $G(t, s), \forall t, s \in(0,1)$, the spectral radius of $L$, denoted by $r(L)$, is positive. The Krein-Rutman theorem [13] then asserts that there are $p \in P \backslash\{0\}$ and $w \in L(0,1) \backslash\{0\}$ such that

$$
\begin{gathered}
\int_{0}^{1} G(t, s) p(s) \mathrm{d} s=r(L) p(t) \\
\int_{0}^{1} G(t, s) w(t) \mathrm{d} t=r(L) w(s) \\
\int_{0}^{1} w(t) \mathrm{d} t=1
\end{gathered}
$$

Denote $\delta:=\int_{0}^{1} \gamma(t) w(t) \mathrm{d} t>0$ and $P_{0}:=\left\{u \in P: \int_{0}^{1} u(t) w(t)\right.$ $\mathrm{d} t \geq \delta\|u\|\}$.

Lemma 4. One has $L(P) \subset P_{0}$.

Proof. Suppose that $u \in P$, then

$$
\begin{aligned}
& \int_{0}^{1}(L u)(t) w(t) \mathrm{d} t \\
& =\int_{0}^{1} \int_{0}^{1} G(t, s) u(s) \mathrm{d} s w(t) \mathrm{d} t \\
& \geq \int_{0}^{1} \gamma(t) w(t) \mathrm{d} t \int_{0}^{1} G(\tau, s) u(s) \mathrm{d} s=\delta(L u)(\tau),
\end{aligned}
$$

for all $\tau \in[0,1]$ and thus $\int_{0}^{1}(L u)(t) w(t) \mathrm{d} t \geq \delta\|L u\|$. This completes the proof.

Lemma 5 (see [14]). Let $E$ be a real Banach space and $\Omega \subset E$ a bounded open set with $0 \in \Omega$. Suppose that $A: \bar{\Omega} \rightarrow E$ is a completely continuous operator. If there is $y_{0} \in E \backslash\{0\}$ such that $u \neq A u+\lambda y_{0}, \forall u \in \partial \Omega, \lambda \geq 0$, then $\operatorname{deg}(I-A, \Omega, 0)=0$, where deg stands for the Leray-Schauder topological degree in E.

\section{Main Results}

Let $\lambda_{1}^{-1}:=r(L)$. We now list our assumptions on $f$.

(H3) $\liminf _{u \rightarrow+\infty}(f(t, u) / u)>\lambda_{1}$ and $\limsup _{u \rightarrow-\infty}$ $(f(t, u) / u)<\lambda_{1}$ uniformly in $t \in[0,1]$.

(H4) There is a positive number $a_{1} \in(0, \delta /(r(L)(\delta+$ $\left.\left.\sum_{k=1}^{m} w\left(t_{k}\right)\right)\right)$ ) such that

$$
\limsup _{u \rightarrow 0} \frac{|f(t, u)|}{|u|}<a_{1}, \quad \limsup _{u \rightarrow 0} \frac{I_{k}(u)}{|u|}<a_{1}
$$

uniformly in $t \in[0,1]$.

(H5) $\liminf _{u \rightarrow 0^{+}}(f(t, u) / u)>\lambda_{1}$ and $\limsup _{u \rightarrow 0^{-}}$ $(f(t, u) / u)<\lambda_{1}$ uniformly in $t \in[0,1]$.
(H6) For $a_{1}$ in (H4), assume that

$$
\begin{array}{r}
\limsup _{u \rightarrow \infty} \frac{|f(t, u)|}{|u|}<a_{1}, \quad \limsup _{u \rightarrow \infty} \frac{I_{k}(u)}{|u|}<a_{1} \\
\text { uniformly in } t \in[0,1] .
\end{array}
$$

Two Examples. Let $\alpha(t)=t, \beta(t)=0$, for all $t \in[0,1]$. Then $\kappa_{1}=1 / n, \kappa_{2}=1 / n, \kappa_{3}=0, \kappa_{4}=1$, and $(\mathrm{H} 2)$ holds true.

(1) Let $f(t, u)=b_{1} u+u^{2}+u^{4}$, where $b_{1} \in\left(0, a_{1}\right)$. Then $f$ satisfies (H1). Moreover, we obtain

$$
\begin{array}{r}
\liminf _{u \rightarrow+\infty} \frac{f(t, u)}{u}=\liminf _{u \rightarrow+\infty}\left(b_{1}+u+u^{3}\right)=+\infty>\lambda_{1}, \\
\text { uniformly in } t \in[0,1],
\end{array}
$$

$$
\begin{array}{r}
\limsup _{u \rightarrow-\infty} \frac{f(t, u)}{u}=\limsup _{u \rightarrow-\infty}\left(b_{1}+u+u^{3}\right)=-\infty<\lambda_{1}, \\
\text { uniformly in } t \in[0,1],
\end{array}
$$

$$
\begin{array}{r}
\limsup _{u \rightarrow 0} \frac{|f(t, u)|}{|u|}=\limsup _{u \rightarrow 0}\left|b_{1}+u+u^{3}\right|=b_{1}<a_{1} \\
\text { uniformly in } t \in[0,1] .
\end{array}
$$

So, $f$ satisfies $(\mathrm{H} 3)$ and $(\mathrm{H} 4)$. Also, let $I_{k}(u)=u^{4}$. Then $I_{k}$ satisfies $(\mathrm{H} 1)$ and

$$
\limsup _{u \rightarrow 0} \frac{I_{k}(u)}{|u|}=\limsup _{u \rightarrow 0}|u|^{3}=0<a_{1} .
$$

Hence, $I_{k}$ satisfies (H4). Consequently, (H1), (H3), and (H4) hold true.

(2) Let $f(t, u)=b_{2} u+2 u^{2 / 3}+3 u^{4 / 5}, I_{k}(u)=\sqrt{|u|}$, where $b_{2} \in\left(0, a_{1}\right)$. Then

$\liminf _{u \rightarrow 0^{+}} \frac{f(t, u)}{u}=\liminf _{u \rightarrow 0^{+}}\left(b_{2}+2 u^{-1 / 3}+3 u^{-1 / 5}\right)=+\infty>\lambda_{1}$ uniformly in $t \in[0,1]$,

$\limsup _{u \rightarrow 0^{-}} \frac{f(t, u)}{u}=\limsup _{u \rightarrow 0^{-}}\left(b_{2}+2 u^{-1 / 3}+3 u^{-1 / 5}\right)=-\infty<\lambda_{1}$ uniformly in $t \in[0,1]$.

Moreover,

$$
\begin{gathered}
\limsup _{u \rightarrow \infty} \frac{|f(t, u)|}{|u|}=\limsup _{u \rightarrow \infty}\left|b_{2}+2 u^{-1 / 3}+3 u^{-1 / 5}\right|=b_{2}<a_{1} \\
\text { uniformly in } t \in[0,1], \\
\limsup _{u \rightarrow \infty} \frac{I_{k}(u)}{|u|}=\limsup _{u \rightarrow \infty} \frac{1}{\sqrt{|u|}}=0<a_{1} .
\end{gathered}
$$

So, (H1), (H5), (H6) hold. 
Theorem 6. Assume that (H1)-(H4) hold, and (1) has at least one nontrivial solution.

Proof. (H3) implies that there are $\varepsilon \in\left(0, \lambda_{1}\right)$ and $c_{1}>0$ such that $f(t, u) \geq\left(\lambda_{1}+\varepsilon\right) u-c_{1}, u \geq 0, t \in[0,1]$ and $f(t, u) \geq$ $\left(\lambda_{1}-\varepsilon\right) u-c_{1}, u \leq 0, t \in[0,1]$. Note that if $(t, u) \in[0,1] \times$ $[0,+\infty), f(t, u) \geq\left(\lambda_{1}+\varepsilon\right) u-c_{1} \geq\left(\lambda_{1}-\varepsilon\right) u-c_{1}$, and if $(t, u) \in[0,1] \times(-\infty, 0], f(t, u) \geq\left(\lambda_{1}-\varepsilon\right) u-c_{1} \geq\left(\lambda_{1}+\varepsilon\right) u-c_{1}$. That is, we have

$$
\begin{array}{ll}
f(t, u) \geq\left(\lambda_{1}+\varepsilon\right) u-c_{1}, & \forall u \in \mathbb{R}, t \in[0,1], \\
f(t, u) \geq\left(\lambda_{1}-\varepsilon\right) u-c_{1}, & \forall u \in \mathbb{R}, t \in[0,1] .
\end{array}
$$

Let $\mathscr{M}_{1}:=\{u \in E: u=A u+\lambda p, \lambda \geq 0\}$, where $p \in P \backslash\{0\}$ is given by (12). We claim that $\mathscr{M}_{1}$ is bounded in $E$. Indeed, if $u_{0} \in \mathscr{M}_{1}$, there is $\lambda_{0} \geq 0$ such that

$$
\begin{aligned}
u_{0}(t)= & \left(A u_{0}\right)(t)+\lambda_{0} p(t)=\int_{0}^{1} G(t, s) f\left(s, u_{0}(s)\right) \mathrm{d} s \\
& +\sum_{k=1}^{m} G\left(t, t_{k}\right) I_{k}\left(u_{0}\left(t_{k}\right)\right)+\lambda_{0} p(t) \\
\geq & \int_{0}^{1} G(t, s) f\left(s, u_{0}(s)\right) \mathrm{d} s .
\end{aligned}
$$

Combining this with (20) yields

$$
u_{0}(t) \geq\left(\lambda_{1}+\varepsilon\right)\left(L u_{0}\right)(t)-c_{1}(L \mathbf{1})(t),
$$

where $\mathbf{1}$ stands for the constant function $\mathbf{1}(t) \equiv 1$. Multiply (23) by $w(t)$ on both sides and integrate over $[0,1]$ and use (12) to obtain $\int_{0}^{1} u_{0}(t) w(t) \mathrm{d} t \geq(1+\varepsilon r(L)) \int_{0}^{1} u_{0}(t) w(t) \mathrm{d} t-$ $c_{1} r(L)$, and thus $\int_{0}^{1} u_{0}(t) w(t) \mathrm{d} t \leq \varepsilon^{-1} c_{1}$. Note that (21) holds. Define the Nemytskii operator $F: E \rightarrow E$ by $(F u)(t):=$ $f(t, u(t))$. Now (22) is equivalent to

$$
\begin{aligned}
u_{0}(t) & -\left(\lambda_{1}-\varepsilon\right)\left(L u_{0}\right)(t)+c_{1}(L \mathbf{1})(t) \\
= & \left(A u_{0}\right)(t)-\left(\lambda_{1}-\varepsilon\right)\left(L u_{0}\right)(t)+c_{1}(L \mathbf{1})(t)+\lambda_{0} p(t) \\
= & \int_{0}^{1} G(t, s)\left[f\left(s, u_{0}(s)\right)-\left(\lambda_{1}-\varepsilon\right) u_{0}(s)+c_{1}\right] \mathrm{d} s \\
& +\sum_{k=1}^{m} G\left(t, t_{k}\right) I_{k}\left(u_{0}\left(t_{k}\right)\right)+\lambda_{0} p(t) \\
= & \left(L\left[F u_{0}-\left(\lambda_{1}-\varepsilon\right) u_{0}+c_{1} \mathbf{1}\right]\right)(t) \\
& +\sum_{k=1}^{m} G\left(t, t_{k}\right) I_{k}\left(u_{0}\left(t_{k}\right)\right)+\lambda_{0} p(t) .
\end{aligned}
$$

Lemma 4 implies that $u_{0}-\left(\lambda_{1}-\varepsilon\right) L u_{0}+c_{1} L \mathbf{1} \in P_{0}$. Therefore,

$$
\begin{aligned}
& \left\|u_{0}-\left(\lambda_{1}-\varepsilon\right) L u_{0}+c_{1} L \mathbf{1}\right\| \\
& \quad \leq \delta^{-1} \int_{0}^{1}\left[u_{0}(t)-\left(\lambda_{1}-\varepsilon\right)\left(L u_{0}\right)(t)+c_{1}(L \mathbf{1})(t)\right] w(t) \mathrm{d} t \\
& \quad \leq \varepsilon \delta^{-1} r(L) \int_{0}^{1} u_{0}(t) w(t) \mathrm{d} t+c_{1} \delta^{-1} r(L) \leq 2 c_{1} \delta^{-1} r(L) .
\end{aligned}
$$

Since $\left(\lambda_{1}-\varepsilon\right) r(L)<1$, the operator $I-\left(\lambda_{1}-\varepsilon\right) L$ has the bounded inverse operator $\left(I-\left(\lambda_{1}-\varepsilon\right) L\right)^{-1}$. Therefore, there is $\mathcal{N}_{1}>0$ such that $\|u\| \leq \mathcal{N}_{1}, \forall u \in \mathscr{M}_{1}$. This proves the boundedness of $\mathscr{M}_{1}$. For each $R>\sup _{u \in \mathscr{M}_{1}}\|u\|$, we have $u \neq A u+\lambda p, \forall u \in \partial B_{R}, \lambda \geq 0$, which leads to

$$
\operatorname{deg}\left(I-A, B_{R}, 0\right)=0 .
$$

On the other hand, (H4) implies that there is a $r>0$ such that $|f(t, u)| \leq a_{1}|u|, I_{k}(u) \leq a_{1}|u|, \forall|u| \leq r, t \in[0,1]$. Notice we may choose $r>0$ so that $r<\sup _{u \in \mathscr{M}_{1}}\|u\|$. Thus we have

$$
\begin{aligned}
& |(A u)(t)| \\
& \leq \int_{0}^{1} G(t, s)|f(s, u(s))| \mathrm{d} s+\sum_{k=1}^{m} G\left(t, t_{k}\right) I_{k}\left(u\left(t_{k}\right)\right) \\
& \leq a_{1}\left[\int_{0}^{1} G(t, s)|u(s)| \mathrm{d} s+\sum_{k=1}^{m} G\left(t, t_{k}\right)\left|u\left(t_{k}\right)\right|\right]
\end{aligned}
$$

for all $u \in \bar{B}_{r}$ and $t \in[0,1]$. We claim that

$$
u \neq \mu A u, \quad \forall u \in \partial B_{r}, \mu \in[0,1] .
$$

Suppose the contrary. Then there are $u_{0} \in \partial B_{r}$ and $\mu_{0} \in[0,1]$ such that $u_{0}=\mu_{0} A u_{0}$. Let $v(t)=\left|u_{0}(t)\right|$. Then $v \in P$ and

$$
v(t) \leq a_{1}\left[\int_{0}^{1} G(t, s) v(s) \mathrm{d} s+\sum_{k=1}^{m} G\left(t, t_{k}\right) v\left(t_{k}\right)\right] .
$$

Multiply (29) by $w(t)$ on both sides and integrate over $[0,1]$ and use (12) to obtain

$$
\begin{aligned}
\int_{0}^{1} v(t) w(t) \mathrm{d} t \leq & a_{1} r(L) \int_{0}^{1} v(t) w(t) \mathrm{d} t \\
& +a_{1} r(L) \sum_{k=1}^{m} v\left(t_{k}\right) w\left(t_{k}\right),
\end{aligned}
$$

and then by Lemma 4 ,

$$
\begin{aligned}
\delta\left(1-a_{1} r(L)\right)\|v\| & \leq\left(1-a_{1} r(L)\right) \int_{0}^{1} v(t) w(t) \mathrm{d} t \\
& \leq a_{1} r(L) \sum_{k=1}^{m} v\left(t_{k}\right) w\left(t_{k}\right) \\
& \leq a_{1} r(L)\|v\| \sum_{k=1}^{m} w\left(t_{k}\right),
\end{aligned}
$$


That is, $a_{1} r(L)\left(\delta+\sum_{k=1}^{m} w\left(t_{k}\right)\right) \geq \delta$, which contracts to $a_{1} \in$ $\left(0, \delta /\left(r(L)\left(\delta+\sum_{k=1}^{m} w\left(t_{k}\right)\right)\right)\right)$. Consequently, $\|v\|=0$, and thus $v=0 \Rightarrow u_{0} \equiv 0$. This contradicts to $u_{0} \in \partial B_{r}$. As a result of this, (28) holds true. Hence $I$ and $I-A$ are homotopic on $\partial B_{r}$. The homotopy invariance of topological degree implies that $\operatorname{deg}\left(I-A, B_{r}, 0\right)=1$. This and (26) together imply that $\operatorname{deg}\left(I-A, B_{R} \backslash \bar{B}_{r}, 0\right)=\operatorname{deg}\left(I-A, B_{R}, 0\right)-\operatorname{deg}\left(I-A, B_{r}, 0\right)=$ -1 . Therefore the operator $A$ has at least one point in $B_{R} \mid$ $\bar{B}_{r}$. Equivalently, (1) has at least one nontrivial solution. This completes the proof.

Theorem 7. Assume that (H1), (H2), (H5), and (H6) hold; (1) has at least one nontrivial solution.

Proof. (H5) implies there are $\varepsilon \in\left(0, \lambda_{1}\right)$ and $r>0$ such that $f(t, u) \geq\left(\lambda_{1}+\varepsilon\right) u, u \in[0, r], t \in[0,1]$, and $f(t, u) \geq\left(\lambda_{1}-\varepsilon\right) u$, $u \in[-r, 0], t \in[0,1]$. Note that if $(t, u) \in[0,1] \times[0, r]$, $f(t, u) \geq\left(\lambda_{1}+\varepsilon\right) u \geq\left(\lambda_{1}-\varepsilon\right) u$, and if $(t, u) \in[0,1] \times[-r, 0]$, $f(t, u) \geq\left(\lambda_{1}-\varepsilon\right) u \geq\left(\lambda_{1}+\varepsilon\right) u$. That is, we have

$$
\begin{array}{ll}
f(t, u) \geq\left(\lambda_{1}+\varepsilon\right) u & \forall u \in[-r, r], t \in[0,1], \\
f(t, u) \geq\left(\lambda_{1}-\varepsilon\right) u, & \forall u \in[-r, r], t \in[0,1] .
\end{array}
$$

Nowadays, we claim

$$
u \neq A u+\lambda p, \quad \forall u \in \partial B_{r}, \quad \lambda \geq 0,
$$

where $p \in P \backslash\{0\}$ is given by (12). Indeed, if the claim is false, then there are $u_{0} \in \partial B_{r}$ and $\lambda_{0} \geq 0$ such that

$$
\begin{aligned}
u_{0}(t)= & \left(A u_{0}\right)(t)+\lambda_{0} p(t) \\
= & \int_{0}^{1} G(t, s) f\left(s, u_{0}(s)\right) \mathrm{d} s \\
& +\sum_{k=1}^{m} G\left(t, t_{k}\right) I_{k}\left(u_{0}\left(t_{k}\right)\right)+\lambda_{0} p(t) \\
\geq & \int_{0}^{1} G(t, s) f\left(s, u_{0}(s)\right) \mathrm{d} s .
\end{aligned}
$$

Combining this with (32) yields

$$
u_{0}(t) \geq\left(\lambda_{1}+\varepsilon\right)\left(L u_{0}\right)(t) .
$$

Multiply (36) by $w(t)$ and integrate over $[0,1]$ and use (12) to obtain $\int_{0}^{1} u_{0}(t) w(t) \mathrm{d} t \geq(1+\varepsilon r(L)) \int_{0}^{1} u_{0}(t) w(t) \mathrm{d} t$, and thus $\int_{0}^{1} u_{0}(t) w(t) \mathrm{d} t \leq 0$. Note that (33) holds, and then we find

$$
\begin{aligned}
u_{0}(t)- & \left(\lambda_{1}-\varepsilon\right)\left(L u_{0}\right)(t) \\
= & \left(A u_{0}\right)(t)-\left(\lambda_{1}-\varepsilon\right)\left(L u_{0}\right)(t)+\lambda_{0} p(t) \\
= & \left(L\left[F u_{0}-\left(\lambda_{1}-\varepsilon\right) u_{0}\right]\right)(t) \\
& +\sum_{k=1}^{m} G\left(t, t_{k}\right) I_{k}\left(u_{0}\left(t_{k}\right)\right)+\lambda_{0} p(t) .
\end{aligned}
$$

Lemma 4 implies that $u_{0}-\left(\lambda_{1}-\varepsilon\right) L u_{0} \in P_{0}$. Therefore, $\left\|u_{0}-\left(\lambda_{1}-\varepsilon\right) L u_{0}\right\| \leq \delta^{-1} \int_{0}^{1}\left[u_{0}(t)-\left(\lambda_{1}-\varepsilon\right)\left(L u_{0}\right)(t)\right] w(t) \mathrm{d} t \leq$ $\varepsilon \delta^{-1} r(L) \int_{0}^{1} u_{0}(t) w(t) \mathrm{d} t \leq 0$. Notice that $\left(\lambda_{1}-\varepsilon\right) r(L)<1$. We have $u_{0}=0$, contradicting $u_{0} \in \partial B_{r}$. As a result of this, (34) is true. Invoking Lemma 5 gives

$$
\operatorname{deg}\left(I-A, B_{r}, 0\right)=0 .
$$

On the other hand, (H6) implies that there is $c_{2}>0$ such that

$$
\begin{array}{r}
|f(t, u)| \leq a_{1}|u|+c_{2}, I_{k}(u) \leq a_{1}|u|+c_{2}, \\
\forall u \in \mathbb{R}, t \in[0,1] .
\end{array}
$$

Let $\mathscr{M}_{2}:=\{u \in E: u=\lambda A u, \forall \lambda \in[0,1]\}$. We shall now show that $\mathscr{M}_{2}$ is bounded in $E$. Indeed, if $u_{0} \in \mathscr{M}_{2}$, then $u_{0}=$ $\lambda_{0} A u_{0}$ for some $\lambda_{0} \in[0,1]$. In view of (39), let $v(t)=\left|u_{0}(t)\right|$, we have $v \in P$ and

$$
\begin{aligned}
v(t) \leq & a_{1}\left[\int_{0}^{1} G(t, s) v(s) \mathrm{d} s+\sum_{k=1}^{m} G\left(t, t_{k}\right) v\left(t_{k}\right)\right] \\
+ & c_{2}\left[\int_{0}^{1} G(t, s) \mathrm{d} s+\sum_{k=1}^{m} G\left(t, t_{k}\right)\right] .
\end{aligned}
$$

Multiply (40) by $w(t)$ on both sides and integrate over $[0,1]$ and use (12) to obtain

$$
\begin{aligned}
\int_{0}^{1} v(t) w(t) \mathrm{d} t \leq & a_{1} r(L) \int_{0}^{1} v(t) w(t) \mathrm{d} t \\
& +a_{1} r(L) \sum_{k=1}^{m} v\left(t_{k}\right) w\left(t_{k}\right) \\
& +c_{2} r(L)\left(1+\sum_{k=1}^{m} w\left(t_{k}\right)\right),
\end{aligned}
$$

and then by Lemma 4,

$$
\begin{aligned}
\delta\left(1-a_{1} r(L)\right)\|v\| \leq & \left(1-a_{1} r(L)\right) \int_{0}^{1} v(t) w(t) \mathrm{d} t \\
\leq & a_{1} r(L) \sum_{k=1}^{m} v\left(t_{k}\right) w\left(t_{k}\right) \\
& +c_{2} r(L)\left(1+\sum_{k=1}^{m} w\left(t_{k}\right)\right) \\
\leq & a_{1} r(L)\|v\| \sum_{k=1}^{m} w\left(t_{k}\right) \\
& +c_{2} r(L)\left(1+\sum_{k=1}^{m} w\left(t_{k}\right)\right) .
\end{aligned}
$$

Hence, $\|v\| \leq\left(c_{2} r(L)\left(1+\sum_{k=1}^{m} w\left(t_{k}\right)\right)\right) /\left(\delta\left(1-a_{1} r(L)\right)-\right.$ $\left.a_{1} r(L) \sum_{k=1}^{m} w\left(t_{k}\right)\right)$. This proves the boundedness of $\mathscr{M}_{2}$. Choosing $R>\sup _{u \in \mathscr{M}_{2}}\|u\|$ and $R>r$, we have $u \neq \lambda A u, \forall u \in$ $\partial B_{R}, \lambda \in[0,1]$. The homotopy invariance of Leray-Schauder topological degree implies that $\operatorname{deg}\left(I-A, B_{R}, 0\right)=1$. Combining this with (38) we get $\operatorname{deg}\left(I-A, B_{R} \backslash \bar{B}_{r}, 0\right)=$ $\operatorname{deg}\left(I-A, B_{R}, 0\right)-\operatorname{deg}\left(I-A, B_{r}, 0\right)=1$. Therefore the operator $A$ has at least one point in $B_{R} \backslash \bar{B}_{r}$. Equivalently, (1) has at least one nontrivial solution. This completes the proof. 


\section{Conflict of Interests}

The authors declare that there is no conflict of interests regarding the publication of this paper.

\section{Acknowledgments}

The authors are supported by the NNSF-China (11371117), Shandong Provincial Natural Science Foundation (ZR2013AM009), GIIFSDU (yzc12063), and IIFSDU (2012TS020).

\section{References}

[1] D. Guo, "Positive solutions of an infinite boundary value problem for $n$ th-order nonlinear impulsive singular integrodifferential equations in Banach spaces," Nonlinear Analysis, Theory, Methods and Applications, vol. 70, no. 5, pp. 2078-2090, 2009.

[2] D. Guo, "Multiple positive solutions for first order impulsive superlinear integro-differential equations on the half line," Acta Mathematica Scientia, vol. 31, no. 3, pp. 1167-1178, 2011.

[3] D. Guo, "Multiple positive solutions for first order impulsive singular integro-differential equations on the half line," Acta Mathematica Scientia, vol. 32, no. 6, pp. 2176-2190, 2012.

[4] H. Zhang, L. Liu, and Y. Wu, "Positive solutions for $n$ th-order nonlinear impulsive singular integro-differential equations on infinite intervals in Banach spaces," Nonlinear Analysis, Theory, Methods and Applications, vol. 70, no. 2, pp. 772-787, 2009.

[5] Y. Xu and H. Zhang, "Positive solutions of an infinite boundary value problem for $n$ th-order nonlinear impulsive singular integro-differential equations in Banach spaces," Applied Mathematics and Computation, vol. 218, no. 9, pp. 5806-5818, 2012.

[6] B. Ahmad and A. Alsaedi, "Existence of solutions for antiperiodic boundary value problems of nonlinear impulsive functional integro-differential equations of mixed type," Nonlinear Analysis, vol. 3, no. 4, pp. 501-509, 2009.

[7] B. Ahmad and S. Sivasundaram, "Existence of solutions for impulsive integral boundary value problems of fractional order," Nonlinear Analysis, vol. 4, no. 1, pp. 134-141, 2010.

[8] X. Zhang, X. Yang, and W. Ge, "Positive solutions of $n$ th-order impulsive boundary value problems with integral boundary conditions in Banach spaces," Nonlinear Analysis, Theory, Methods and Applications, vol. 71, no. 12, pp. 5930-5945, 2009.

[9] M. Feng, X. Zhang, and X. Yang, "Positive solutions of $n$ thorder nonlinear impulsive differen- tial equation with nonlocal boundary conditions," Boundary Value Problems, vol. 2011, Article ID 456426, 19 pages, 2011.

[10] M. Feng and H. Pang, "A class of three-point boundary-value problems for second-order impulsive integro-differential equations in Banach spaces," Nonlinear Analysis, Theory, Methods and Applications, vol. 70, no. 1, pp. 64-82, 2009.

[11] Z. Yang, "Existence of nontrivial solutions for a nonlinear Sturm-Liouville problem with integral boundary conditions," Nonlinear Analysis, Theory, Methods and Applications, vol. 68, no. 1, pp. 216-225, 2008.

[12] J. Xu and Z. Yang, "Positive soltuions for a system of $n$ thorder nonlinear boundary value problem," Electronic Journal of Qualitative Theory of Differential Equations, vol. 4, pp. 1-16, 2011.
[13] M. Krein and M. Rutman, "Linear operators leaving invariant a cone in a Banach space," American Mathematical Society Translations, vol. 10, no. 1, pp. 199-325, 1962.

[14] D. Guo and V. Lakshmikantham, Nonlinear Problems in Abstract Cones, Academic Press, Boston, Mass, USA, 1988. 


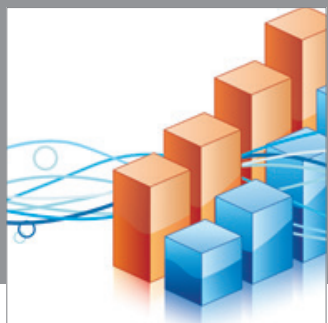

Advances in

Operations Research

mansans

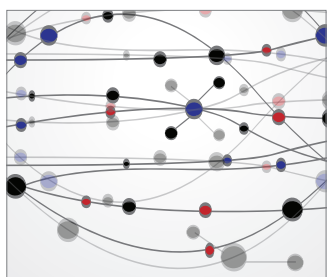

The Scientific World Journal
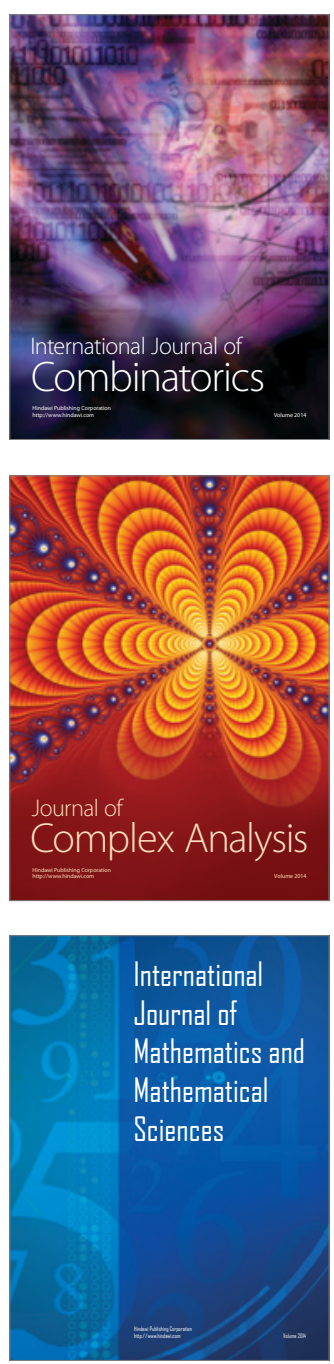
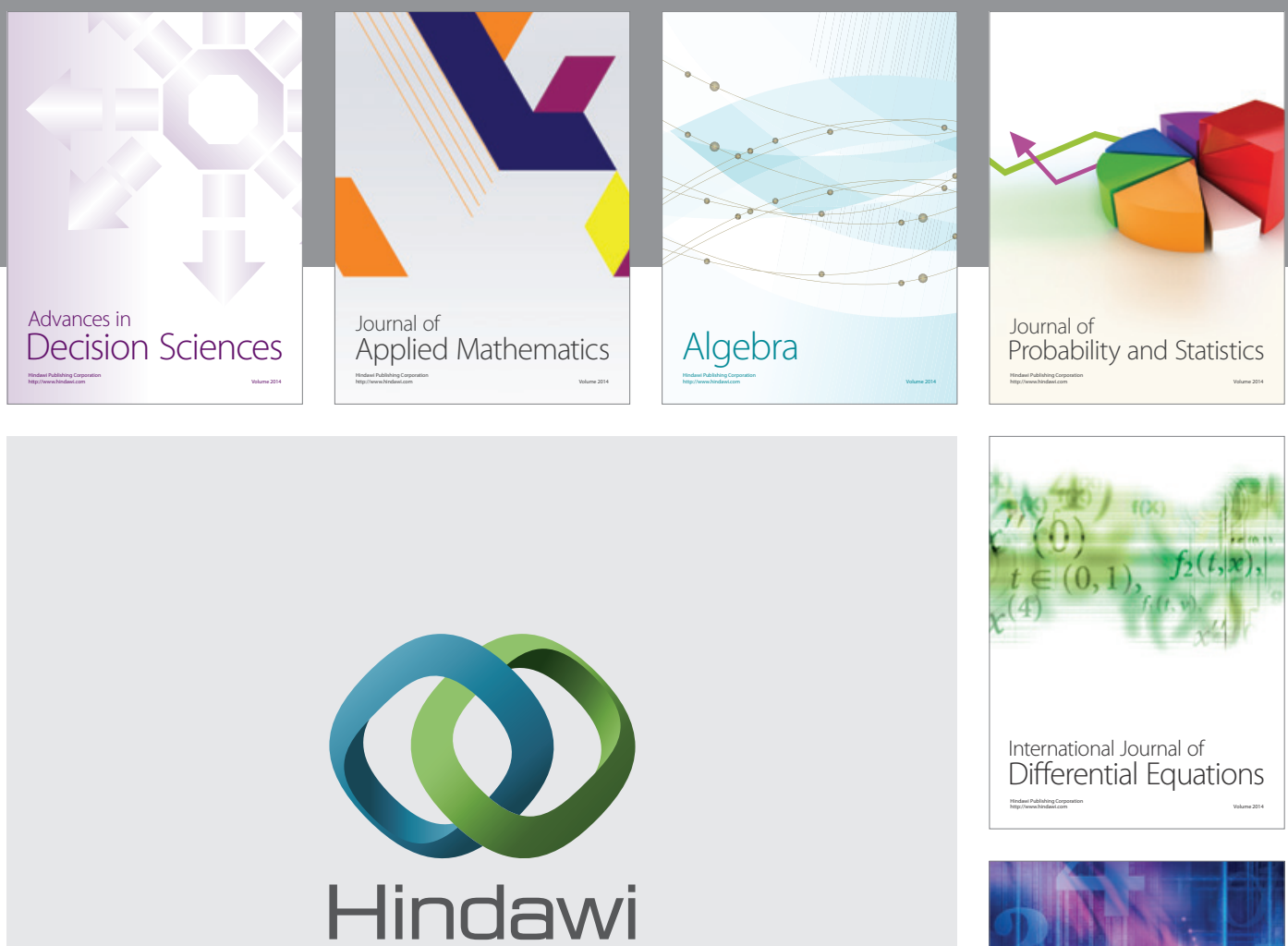

Submit your manuscripts at http://www.hindawi.com
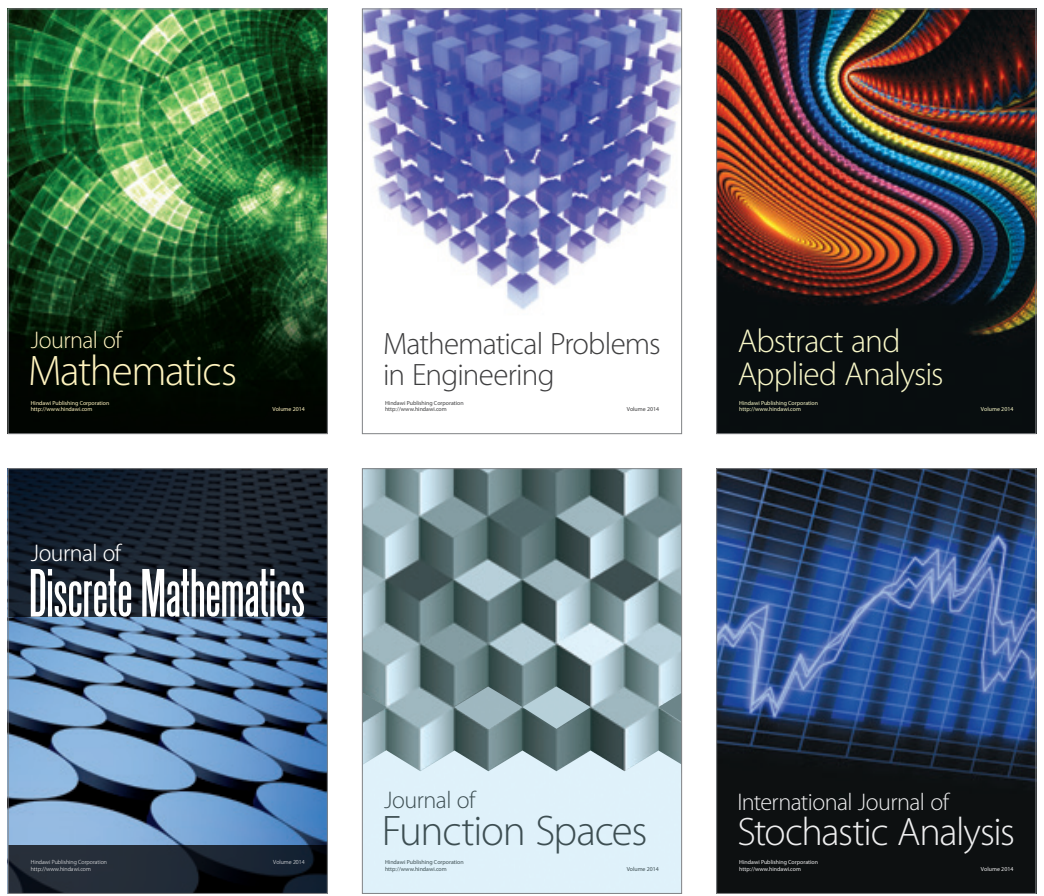

Journal of

Function Spaces

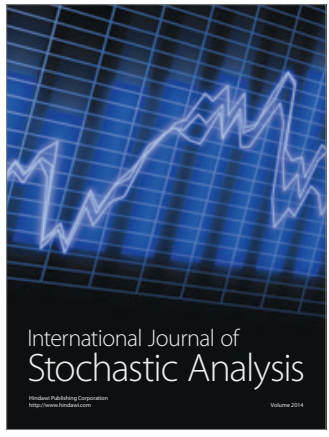

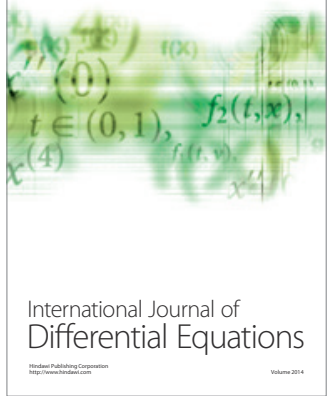
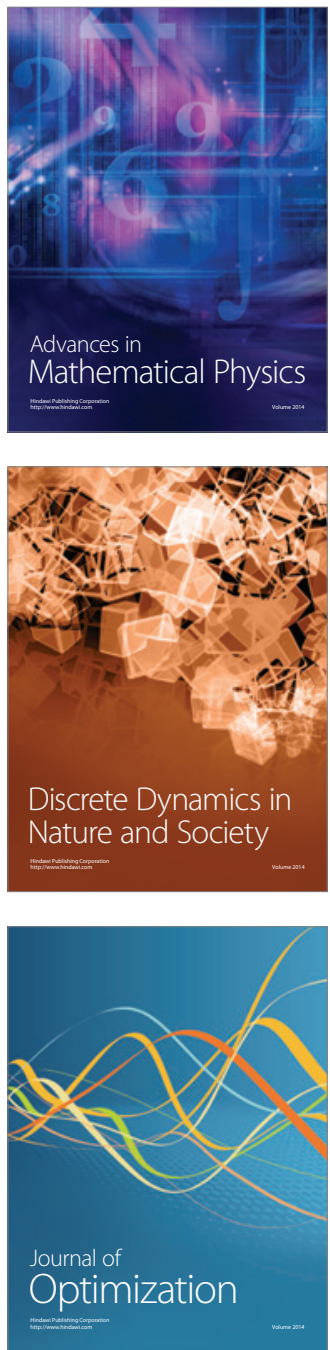